\title{
Preface: Special Issue of Selected Extended Papers of CADE 2019
}

\author{
Pascal Fontaine ${ }^{1}$
}

Published online: 18 June 2021

(c) The Author(s), under exclusive licence to Springer Nature B.V. 2021

This special issue of the Journal of Automated Reasoning is dedicated to selected papers presented at the 27th International Conference on Automated Deduction (CADE-27) [1], held at the Universidade Federal do Rio Grande do Norte, Natal, Brazil between August 23 and 30, 2019. CADE is the major forum for the presentation of research in all aspects of automated deduction, including foundations, applications, implementations, and practical experience. The six papers selected for the special issue (out of 34 papers presented at the conference) underwent a new and thorough reviewing and revision process, in accordance with the high standards of $J A R$.

One of the cornerstones of automated deduction for first-order logic is the superposition calculus. In their paper "Superposition with Lambdas", Alexander Bentkamp, Jasmin Blanchette, Sophie Tourret, Petar Vukmirović, and Uwe Waldmann lift this calculus to higherorder reasoning.

In their work "Model Completeness, Uniform Interpolants and Superposition Calculus", Diego Calvanese, Silvio Ghilardi, Alessandro Gianola, Marco Montali, and Andrey Rivkin investigate covers and model completions as means for model checking of transition systems, and particularly of simple artifact systems, where the signature and theory relate to database schemata.

The paper "Automata Terms in a Lazy WSkS Decision Procedure", by Vojtěch Havlena, Luká Holík, Ondřej Lengál, and Tomáš Vojnar, introduces an elegant lazy decision procedure for the logic WS $k$ S, mixing automata based and symbolic techniques. The conference version of this paper was awarded the CADE-27 best paper award.

The last two decades have seen great improvements in reasoning modulo theories, notably for bit-vector reasoning. Generally, a fixed bit-vector width is assumed. In their paper "Towards Satisfiability Modulo Parametric Bit-vectors", Aina Niemetz, Mathias Preiner, Andrew Reynolds, Yoni Zohar, Clark Barrett, and Cesare Tinelli pave the way for reasoning on bit-vectors independently of the width.

Automated reasoning is also useful as a tool to better understand logic itself. In their paper "Distilling the Requirements of Gödel's Incompleteness Theorems with a Proof Assistant", Andrei Popescu and Dmitriy Traytel present a new formalization of Gödel's incompleteness

$\bowtie \quad$ Pascal Fontaine

Pascal.Fontaine@uliege.be

1 Montefiore Institute, University of Liège, Liège, Belgium 
theorems in the Isabelle proof assistant, with the focus of imposing as weak constraints as possible on the theories considered.

In their work "Optimization Modulo the Theory of Floating-Point Numbers", Patrick Trentin and Roberto Sebastiani explore reasoning modulo theories beyond satisfiability, and design a practical approach for optimization in presence of floating-point constraints.

I am thankful to the authors of the submitted papers and to the programme committee of $C A D E-27$. I am especially grateful to the expert reviewers who agreed to review the papers submitted to this special issue for their diligence and timely effort, particularly in these times where COVID-19 impacted a lot our work and our schedules. Finally, I would like to thank all people at Springer for their support, and in particular Tobias Nipkow, who was the editorin-chief of JAR when this special issue has been initiated and remained in charge of it until publication.

Liège, March 2021

\section{References}

1. 27th International Conference on Automated Deduction (CADE-27). Lecture Notes in Computer Science, vol. 11716. Springer, Berlin (2019)

Publisher's Note Springer Nature remains neutral with regard to jurisdictional claims in published maps and institutional affiliations. 